UFIFT-HEP-09-05

\title{
Summing Planar Open String Loops on a Worldsheet Lattice with Dirichlet and Neumann Boundaries
}

\author{
Charles B. Thorn ${ }^{1}$ \\ School of Natural Sciences, Institute for Advanced Study, Princeton NJ 08540 \\ and \\ Institute for Fundamental Theory \\ Department of Physics, University of Florida, Gainesville FL 32611
}

\begin{abstract}
We extend the lightcone worldsheet lattice approach to string theory, proposed in 1977 by Giles and me, to allow for coincident D-branes. We find a convenient lattice representation of Dirichlet boundary conditions, which the open string coordinates transverse to the D-branes satisfy. We then represent the sum over all planar open string multi-loop diagrams by introducing an Ising spin system on the worldsheet lattice to keep track of the presence or absence of fluctuating boundaries. Finally we discuss a simple mean field treatment of the resulting coupled Ising/coordinate worldsheet system. The interplay between Neumann and Dirichlet boundary conditions leads to a richer phase structure, within this mean field approximation, than that found by Orland for the original system with only Neumann conditions.
\end{abstract}

\footnotetext{
${ }^{1}$ E-mail address: thorn@phys.ufl.edu
} 


\section{Introduction}

The problem of summing planar diagrams is central to many issues in theoretical physics, from the large $N$ approximation to QCD [1] to the relationship of string theory to quantum field theory as exemplified by the AdS/CFT correspondence [2]. In the absence of an analytic solution to this problem, it would be nice to have an approach amenable to numerical simulations. Over 30 years ago, Giles and I proposed a way to digitize interacting bosonic string theory, in its first quantized version, as a sum over histories on a lightcone worldsheet lattice [3] (GT). Although inspired by perturbation theory, the resulting formalism provides a fully non-perturbative dynamics which reproduces the formal ${ }^{2}$ perturbation theory when expanded in powers of the string coupling $g$. The restriction of this sum over histories to planar open string loops should be manageable on a computer.

In recent years, my colleagues and I have constructed an explicit lightcone worldsheet representation of the planar diagrams of a wide range of matrix field theories [6]. These constructions rely on fermionic worldsheet ghosts to cancel the bulk degrees of freedom of each worldsheet coordinate - the worldsheet systems that reproduce field theory diagrams are essentially topological. The ensuing negative signs in the path integrands spell serious difficulties for numerical simulations of such worldsheet path integrals. This problem can be avoided by replacing each field quantum with a finite tension open string, bringing us back to the original GT formalism. Thus we propose that numerical simulations of planar diagram sums be performed in the GT formalism at finite string tension, after which conclusions about field theory can be drawn by study of the infinite tension limit.

All open string coordinates in [3] satisfy free end (Neumann) boundary conditions. It has long been clear that D-branes [7] provide the key for arranging that the infinite tension limit of critical string theory (in 26 or 10 space-time dimensions) yield a quantum field theory in lower dimensional space-time. D-branes are subspaces on which open strings end, meaning that the open string coordinates describing motion perpendicular to the D-branes satisfy Dirichlet boundary conditions. The purpose of this article is to explain how such conditions can be introduced in the GT worldsheet lattice formalism, and to begin to assess, in the context of a simple mean field approximation, their impact on the physics of planar diagram summation.

In addition to bringing in Dirichlet boundaries, the GT lattice formalism also needs to be extended to include Grassmann coordinates in order to describe the Neveu-Schwarz (NS) boson model [8,9], Ramond fermions [10,11], or the superstring [12]. In particular, we have suggested that the even G-parity Neveu-Schwarz open string model with $\mathrm{SU}(N)$ Chan-Paton factors (NS+), which is free of open string tachyons, could be used to establish a string representation of large $N$ QCD $[13,14]$. Since the lightcone worldsheet lattice is tailor made for summing planar diagrams, its extension to cover the NS+ open string model would provide a promising way to sum planar diagrams on a computer. Then study of the $T_{0} \rightarrow \infty$ limit should yield new information about large $N$ QCD.

\footnotetext{
${ }^{2}$ Technically Lorentz covariance in the continuum limit requires counterterms to cancel lattice artifacts that arise from boundary terms in the integration over moduli (see e.g. $[4,5]$ ). Bulk and boundary terms already included in [3] can account for some (perhaps all) of the necessary counterterms.
} 
We conclude this introduction with a brief review of the original GT lattice formalism for bosonic string theory [3]. It starts with a lattice worldsheet path integral for the lightcone quantized free open string [15],

$$
\begin{aligned}
\left\langle\boldsymbol{x}_{f}\left|e^{-T P^{-}}\right| \boldsymbol{x}_{i}\right\rangle & \equiv \int \mathcal{D} \boldsymbol{x} \exp \left\{-\int_{0}^{T} d \tau \int_{0}^{P^{+}} d \sigma \frac{1}{2}\left(\dot{\boldsymbol{x}}^{2}+T_{0}^{2} \boldsymbol{x}^{2}\right)\right\} \\
& \rightarrow \int \prod_{j=1}^{N} \prod_{i=1}^{M} d \boldsymbol{x}_{i}^{j} \exp \left\{-\frac{T_{0}}{2} \sum_{j=0}^{N} \sum_{i=1}^{M}\left(\boldsymbol{x}_{i}^{j+1}-\boldsymbol{x}_{i}^{j}\right)^{2}-V_{\mathrm{N}}^{\text {open }}\left(\boldsymbol{x}_{i}^{j}\right)\right\} \\
V_{\mathrm{N}}^{\text {open }} & =\frac{T_{0}}{2} \sum_{j=1}^{N} \sum_{i=1}^{M-1}\left(\boldsymbol{x}_{i+1}^{j}-\boldsymbol{x}_{i}^{j}\right)^{2}
\end{aligned}
$$

where for simplicity we have taken the discrete unit of $\sigma$ to be $T_{0} a\left(P^{+}=M a T_{0}\right)$, with $a$ the discrete unit of $\tau(T=(N+1) a)$. Then $a$ drops out of the formulae and the continuum limit is simply $M, N \rightarrow \infty$ with $N / M=T_{0} T / P^{+}$fixed. In the above expression, $\boldsymbol{x}_{k}^{0} \equiv \boldsymbol{x}_{k}^{i}$ and $\boldsymbol{x}_{k}^{N+1} \equiv \boldsymbol{x}_{k}^{f}$ are fixed by the initial and final states.

We have written the potential term $V_{\mathrm{N}}$ of the lattice action appropriate to the open string, with Neumann boundary conditions which are automatic consequences of the absence of a "bond" joining the sites $(1, j)$ to the respective sites $(M, j)$. The closed string action would be obtained by simply restoring those bonds.

$$
V^{\text {closed }}\left(\boldsymbol{x}_{i}^{j}\right)=V_{\mathrm{N}}^{\text {open }}\left(\boldsymbol{x}_{i}^{j}\right)+\frac{T_{0}}{2} \sum_{j=1}^{N}\left(\boldsymbol{x}_{1}^{j}-\boldsymbol{x}_{M}^{j}\right)^{2}
$$

More generally, by rearranging the bond patterns in the potential energy term $V$ of the worldsheet action $S$, we can describe any number of closed and open strings. Then interactions among strings can be achieved by summing over histories in which the bond patterns change from time to time [3]. Each appearance or disappearance of a bond is accompanied by a factor of $g$, and each bond interchange by a factor of $g^{2}$.

This general sum over histories would involve wildly nonlocal interactions on the worldsheet, which would surely defeat any attempt at numerical evaluation. However, the numerical prospects are much brighter for the sum over histories corresponding to planar open string multiloop diagrams ${ }^{3}$. In this case the only changes in bond patterns would be the appearance or disappearance of bonds between nearest neighbor sites. Since these changes are all local on the worldsheet, techniques of condensed matter physics and quantum field theory should apply. For instance, Orland [16] has applied the technique of mean field theory to study the physics of this planar diagram summation ${ }^{4}$. He introduced an Ising spin

\footnotetext{
${ }^{3}$ Incorporating an $\mathrm{SU}(N)$ "color" symmetry via Chan-Paton factors, these planar diagrams are singled out by 't Hooft's large $N$ limit [1]. With a canonically normalized gauge coupling $g_{s}$ the limit holds $g_{s}^{2} N$ fixed. To simplify writing we will absorb a factor of $\sqrt{N}$ in our coupling: $g=g_{s} \sqrt{N}$

${ }^{4}$ See [17] for a recent alternative treatment of mean field theory in this context.
} 
variable $s_{i}^{j}= \pm 1$ to represent the two states "on" $(s=+1)$ or "off" $(s=-1)$ of each planar bond. Putting $P_{i}^{j}=\left(1+s_{i}^{j}\right) / 2$, we then have

$$
\begin{aligned}
\left\langle\boldsymbol{x}_{f}\left|e^{-T P^{-}}\right| \boldsymbol{x}_{i}\right\rangle_{\text {Planar }} \rightarrow & \prod_{i, j} \sum_{P_{i}^{j}=0,1} \int \prod_{j=1}^{N} \prod_{i=1}^{M} d \boldsymbol{x}_{i}^{j} \exp \{-S(\boldsymbol{x}, P)\} \\
S(\boldsymbol{x}, P)= & \sum_{j=0}^{N} \sum_{i=1}^{M}\left[\frac{T_{0}}{2}\left(\boldsymbol{x}_{i}^{j+1}-\boldsymbol{x}_{i}^{j}\right)^{2}+\frac{T_{0}}{2} P_{i}^{j}\left(\boldsymbol{x}_{i+1}^{j}-\boldsymbol{x}_{i}^{j}\right)^{2}\right. \\
& \left.+\alpha+\beta\left(1-P_{i}^{j}\right)-\left(P_{i}^{j+1}-P_{i}^{j}\right)^{2} \ln g\right]
\end{aligned}
$$

The terms on the last line account for the coupling constant $g$ and the bulk $(\alpha)$ and boundary $(\beta)$ worldsheet counterterms. Since we have included a fluctuating bond between $i=1$ and $i=M$, this expression describes the planar evolution of a closed string. For the corresponding evolution of an open string, one simply imposes the constraint $P_{M}^{j}=0$.

Although (6) is completely well-defined and finite for fixed $M, N$, it allows for only the two natural counterterms that were introduced in [3], and shown to be necessary at the level of free strings $(g=0)$. Indeed, the lattice evaluation predicts that the closed and open free bosonic string ground state energies have the $M \rightarrow \infty$ behavior

$$
\begin{aligned}
P_{\text {closed }}^{-} & \sim \frac{D-2}{a}\left[\frac{2 M G}{\pi}\right]-\frac{(D-2) \pi T_{0}}{6 P^{+}} \\
P_{\text {open }}^{-} & \sim \frac{D-2}{a}\left[\frac{2 M G}{\pi}-\frac{1}{2} \ln (1+\sqrt{2})\right]-\frac{(D-2) \pi T_{0}}{24 P^{+}}
\end{aligned}
$$

where $G=\sum_{n=0}^{\infty}(-)^{n} /(2 n+1)^{2} \approx 0.9159656$ is Catalan's constant. In [3] we observed that the divergent (and non-Lorentz invariant) terms can be absorbed in $\alpha$ and $\beta$. Choosing

$$
\alpha=-\frac{2 G(D-2)}{\pi}+O\left(g^{2}\right), \quad \beta=\frac{D-2}{2} \ln (1+\sqrt{2})+O\left(g^{2}\right)
$$

gives finite, Lorentz covariant, and correct values for the free string energy spectrum. Although it was not clearly stated in [3], we must expect that both of these counterterm parameters will receive corrections for nonzero coupling $g \neq 0$ in order to maintain Lorentz covariance for $D=26$. Indeed, the one loop corrections in bosonic string theory do contribute to them. An important open problem, not addressed in this article, is to resolve whether these two counterterms suffice to render the loop expansion covariant. If not, any further counterterms must be identified and incorporated into the formalism.

The rest of the article is organized as follows. In Section 2 we present our prescription for handling Dirichlet boundary conditions on the lattice. In Section 3, we represent the sum over planar diagrams, in which some open string coordinates satisfy Neumann boundary conditions and others satisfy Dirichlet boundary conditions, as a sum over Ising spin configurations, where the Ising spin keeps track of the fluctuating boundaries. Then in Section 4, we give our implementation of the mean field approximation to the Ising spin dynamics. Section 5 concludes the article with a discussion of our results and problems for the future. 


\section{Dirichlet Conditions on the Worldsheet Lattice}

For notational clarity we shall use $\boldsymbol{x}$ to describe the string coordinates satisfying Neumann conditions, and we shall use $\boldsymbol{y}$ for the string coordinates satisfying Dirichlet conditions. We first consider a single free string with Dirichlet conditions $\boldsymbol{y}_{0}^{j}=\boldsymbol{y}_{M}^{j}=0$. The simplest way to discretize this string is to use the action:

$$
S^{\prime}=\frac{T_{0}}{2} \sum_{j=0}^{N} \sum_{i=1}^{M-1}\left(\boldsymbol{y}_{i}^{j+1}-\boldsymbol{y}_{i}^{j}\right)^{2}+\frac{T_{0}}{2} \sum_{j=1}^{N}\left(\sum_{i=1}^{M-2}\left(\boldsymbol{y}_{i+1}^{j}-\boldsymbol{y}_{i}^{j}\right)^{2}+\left(\boldsymbol{y}_{1}^{j}\right)^{2}+\left(\boldsymbol{y}_{M-1}^{j}\right)^{2}\right)
$$

Note that $S^{\prime}$ involves only $M-1$ integration variables for each time slice $j$. Now consider how to obtain this action from the closed string action, which involves $M$ integrations for each $j$, as would be necessary in the sum over histories. Then, the replacement

$$
\left(\boldsymbol{y}_{1}^{j}-\boldsymbol{y}_{M}^{j}\right)^{2}+\left(\boldsymbol{y}_{M}^{j}-\boldsymbol{y}_{M-1}^{j}\right)^{2} \rightarrow\left(\boldsymbol{y}_{1}^{j}\right)^{2}+\left(\boldsymbol{y}_{M-1}^{j}\right)^{2},
$$

encounters the problem that the coordinate $\boldsymbol{y}_{M}$ describes a spurious zero frequency mode. An easy fix for this is to include an extra term $T_{0} \sum_{j=1}^{N}\left(\boldsymbol{y}_{M}^{j}\right)^{2}$ in $S_{D}$ :

$$
\begin{aligned}
S_{\mathrm{D}} & \equiv \frac{T_{0}}{2} \sum_{j=0}^{N} \sum_{i=1}^{M}\left(\boldsymbol{y}_{i}^{j+1}-\boldsymbol{y}_{i}^{j}\right)^{2}+V_{\mathrm{D}}\left(\boldsymbol{y}_{i}^{j}\right) \\
V_{\mathrm{D}}\left(\boldsymbol{y}_{i}^{j}\right) & =\frac{T_{0}}{2} \sum_{j=1}^{N}\left\{\sum_{i=1}^{M-2}\left(\boldsymbol{y}_{i+1}^{j}-\boldsymbol{y}_{i}^{j}\right)^{2}+\boldsymbol{y}_{1}^{j 2}+\boldsymbol{y}_{M-1}^{j 2}+2 \boldsymbol{y}_{M}^{j 2}\right\}
\end{aligned}
$$

Then, since the frequency of this added mode is $O(1)$ in lattice units, the mode described by $\boldsymbol{y}_{M}^{j}$ is irrelevant in the continuum limit. But retaining it allows an efficient description of the creation and destruction of Dirichlet boundaries without changes in the number of degrees of freedom.

Before turning to that, we give the explicit evaluation of the path history sum for the propagation of a free Dirichlet string. Define

$$
\begin{aligned}
\alpha_{n} & \equiv 4 \sin ^{2} \frac{n \pi}{2(N+1)}, \quad n=1,2, \ldots, N \\
\beta_{m} & \equiv 4 \sin ^{2} \frac{m \pi}{2 M}, \quad m=0,1, \ldots, M-1
\end{aligned}
$$

which are the respective eigenvalues of the kinetic and potential bilinear forms occurring in $S_{\mathrm{N}}$. The eigenvalues of the potential bilinear form appearing in $S_{\mathrm{D}}$ are the $\beta_{m}, m=$ $1, \ldots, M-1$, plus the eigenvalue 2 for the extra coordinate $\boldsymbol{y}_{M}^{j}$. For economy of writing it is convenient to put $\beta_{M} \equiv 2$ and to define the frequencies

$$
\omega_{m} \equiv 2 \sinh ^{-1} \frac{\sqrt{\beta_{m}}}{2}, \quad \text { for } m=1, \ldots, M
$$


Then the path integral for a Dirichlet string propagating in $d$ dimensions over time $T=$ $(N+1) a$ from $\boldsymbol{y}_{i}=0$ to $\boldsymbol{y}_{f}=0$ using (12) is

$$
\begin{aligned}
\left\langle\mathbf{0}\left|e^{-(N+1) a P^{-}}\right| \mathbf{0}\right\rangle_{D} & =\left\{\left[\frac{T_{0}}{2 \pi}\right]^{M N} \prod_{n=1}^{N} \prod_{m=1}^{M}\left(\alpha_{n}+\beta_{m}\right)\right\}^{-d / 2} \\
& =\left\{\left[\frac{T_{0}}{2 \pi}\right]^{M N} \prod_{m=1}^{M} \frac{\sinh \left((N+1) \omega_{m}\right)}{\sinh \left(\omega_{m}\right)}\right\}^{-d / 2} \\
& \sim e^{-(N+1) E_{M}}\left[\frac{T_{0}(1+\sqrt{2})}{2 \pi}\right]^{M d / 2} \frac{(3 M)^{d / 4}}{\prod_{m=1}^{\infty}\left(1-w^{2 m}\right)^{d / 2}}
\end{aligned}
$$

where we have taken $M$ large in the last line and introduced $w \equiv e^{-(N+1) \pi / M}=e^{-T \pi T_{0} / P^{+}}$. Here,

$$
\begin{aligned}
E_{M} & =\frac{M d}{2} \ln \frac{T_{0}}{2 \pi}+\frac{d}{2} \sum_{m=1}^{M} \omega_{m} \\
& \sim \frac{M d}{2}\left(\frac{4 G}{\pi}+\ln \frac{T_{0}}{2 \pi}\right)+\frac{d}{2} \ln \frac{2+\sqrt{3}}{1+\sqrt{2}}-\frac{\pi}{24 M}+O\left(M^{-2}\right)
\end{aligned}
$$

The first two terms, one linear in $M$ and the other independent of $M$, contribute divergent terms to the continuum $P^{-}=E_{M} / a$ and violate Lorentz invariance. But they can be cancelled by the bulk and boundary counterterms respectively. Here we see explicitly that the effect of the $\boldsymbol{y}_{M}$ mode we added is simply to modify the coefficient $\beta$ of the boundary counterterm. Taking the case of $D-2$ Neumann and 26-D Dirichlet open string coordinates, we see that we should have

$$
\begin{aligned}
\beta & =-\frac{26-D}{2} \ln \frac{2+\sqrt{3}}{1+\sqrt{2}}+\frac{D-2}{2} \ln (1+\sqrt{2})+O\left(g^{2}\right) \\
& =-\frac{26-D}{2} \ln (2+\sqrt{3})+12 \ln (1+\sqrt{2})+O\left(g^{2}\right)
\end{aligned}
$$

For $D=4$ this is $\beta=-3.91+O\left(g^{2}\right)$. Curiously, at $g=0, \beta$ stays negative for $D<10$ and is positive for $D \geq 10$. For $D=10, \beta \approx 0.041+O\left(g^{2}\right)$.

\section{Summing Planar Diagrams}

We turn now to the problem of representing the sum of planar diagrams by introducing the same system of Ising spins $s_{i}^{j}= \pm 1$ or equivalently $P_{i}^{j}=\left(1+s_{i}^{j}\right) / 2=0,1$ used in the case of Neumann conditions [16]. Associate each coordinate $\boldsymbol{y}_{i}^{j}$ with the corresponding $P_{i}^{j}$ and let $P_{i}^{j}=0$ when the Dirichlet condition applies to $\boldsymbol{y}_{i}^{j}$. Then we should write the potential 
term as:

$$
\begin{aligned}
\frac{T_{0}}{2} \sum_{i, j} & {\left[P_{i}^{j} P_{i+1}^{j}\left(\boldsymbol{y}_{i+1}^{j}-\boldsymbol{y}_{i}^{j}\right)^{2}+\left(1-P_{i}^{j}\right) P_{i+1}^{j} \boldsymbol{y}_{i+1}^{j 2}+\left(1-P_{i}^{j}\right) P_{i-1}^{j} \boldsymbol{y}_{i-1}^{j 2}+2\left(1-P_{i}^{j}\right) \boldsymbol{y}_{i}^{j 2}\right] } \\
& =T_{0} \sum_{i, j}\left[\boldsymbol{y}_{i}^{j 2}-P_{i}^{j} P_{i+1}^{j} \boldsymbol{y}_{i}^{j} \cdot \boldsymbol{y}_{i+1}^{j}\right] .
\end{aligned}
$$

Then the lightcone worldsheet action that sums the planar diagrams of Dirichlet open strings would be

$$
S_{\mathrm{D}}=\frac{T_{0}}{2} \sum_{j=0}^{N} \sum_{i=1}^{M}\left(\boldsymbol{y}_{i}^{j+1}-\boldsymbol{y}_{i}^{j}\right)^{2}+T_{0} \sum_{j=1}^{N} \sum_{i=1}^{M}\left(\boldsymbol{y}_{i}^{j 2}-\boldsymbol{y}_{i}^{j} \cdot \boldsymbol{y}_{i+1}^{j} P_{i}^{j} P_{i+1}^{j}\right)
$$

Notice that this implementation of Dirichlet conditions has the feature that if every site is Dirichlet, i.e. $P_{i}^{j}=0$ for all $i, j$, then the system is just $M$ independent oscillators with frequency of $O(1)$, and the continuum limit would show no interesting physics.

In order to describe $D=4$ dimensional physics with a critical string theory in 26 (bosonic) or 10 (Neveu-Schwarz) space-time dimensions, one can, as in the development of the AdS/CFT correspondence [2], introduce a stack of $N$ coincident D3-branes, which are $3+1$ dimensional subspaces on which open strings end. Let us call the 4 coordinates parallel to the D3-branes $x^{\mu}$ and the coordinates perpendicular to the D3-branes $y^{I}$. For the bosonic string $I$ takes 22 values and for the Neveu-Schwarz string it takes on 6 values. The coordinates $x(\sigma, \tau)$ for an open string satisfy Neumann boundary conditions $\partial x / \partial \sigma=0$ whereas the coordinates $y$ satisfy Dirichlet boundary conditions $y^{I}=0$. Then a possible worldsheet lattice set up would be

$$
\begin{aligned}
S= & \sum_{i, j}\left[\alpha+\beta\left(1-P_{i}^{j}\right)\right]-\sum_{i, j}\left(P_{i}^{j+1}-P_{i}^{j}\right)^{2} \ln g+\frac{T_{0}}{2} \sum_{j=0}^{N} \sum_{i=1}^{M}\left[\left(\boldsymbol{x}_{i}^{j+1}-\boldsymbol{x}_{i}^{j}\right)^{2}+\left(\boldsymbol{y}_{i}^{j+1}-\boldsymbol{y}_{i}^{j}\right)^{2}\right] \\
& +\frac{T_{0}}{2} \sum_{j=1}^{N} \sum_{i=1}^{M}\left[\left(\boldsymbol{x}_{i+1}^{j}-\boldsymbol{x}_{i}^{j}\right)^{2} P_{i}^{j}+2 \boldsymbol{y}_{i}^{j 2}-2 \boldsymbol{y}_{i}^{j} \cdot \boldsymbol{y}_{i+1}^{j} P_{i}^{j} P_{i+1}^{j}\right]
\end{aligned}
$$

The sum over planar diagrams is accomplished by summing over all spin configurations $P_{i}^{j}=0,1$. It is worth pointing out the physical situations represented by the extreme spin configurations. If all $P_{i}^{j}=1$ The $x$ 's and $y$ 's appear on an equal footing and represent a single closed string moving in 25 spatial dimensions (for the bosonic string). In the opposite extreme, with all $P_{i}^{j}=0$, the $x$ 's see no potential and represent $M$ free Newtonian particles moving in 2 spatial dimensions, and the $y$ 's represent $M$ such particles bound by a harmonic oscillator potential to the point $\boldsymbol{y}=0$.

The parameters $\alpha$ and $\beta$ characterize the bulk and boundary counterterms respectively. They will depend on $g$ in a way that we do not know a priori. It may well be that counterterms beyond these will be required to ensure Lorentz invariance, though there remains a slender hope that these will suffice. A study of multi-loop corrections in perturbation theory will be needed to resolve this issue. As a working hypothesis we shall assume in this article that only these counterterms play a role. 


\section{Mean Field Theory}

Mean field theory provides a simple method to understand the physics of large systems, although it can be misleading especially near critical points. A convenient framework for applying mean field theory to our spin system begins with the addition of a source term $\sum_{i j} \kappa_{j}^{i} P_{i}^{j}$ to the action $S$. Then, writing the path integral in the presence of $\kappa$ as $\mathcal{Z}(\kappa) \equiv$ $e^{-\mathcal{F}(\kappa)}$, the expectations and correlators of the $P$ 's can be obtained as derivatives of $\mathcal{F}$ with respect to the $\kappa$ 's. Defining

$$
\phi_{i}^{j} \equiv\left\langle P_{i}^{j}\right\rangle=\frac{\partial \mathcal{F}}{\partial \kappa_{j}^{i}},
$$

it follows that the Legendre transform $\mathcal{A}(\phi) \equiv \mathcal{F}-\sum_{i j} \kappa_{j}^{i} \phi_{i}^{j}$ satisfies

$$
\frac{\partial \mathcal{A}}{\partial \phi_{i}^{j}}=-\kappa_{j}^{i} .
$$

Thus the possible values of $\phi$ are stationary points of the effective action $\mathcal{A}$ in the absence of sources.

Up to this point no approximations have been made. The mean field approximation consists in replacing the coefficients of the coordinate terms in the action by their expectation values:

$$
\begin{gathered}
P_{i}^{j} \rightarrow\left\langle P_{i}^{j}\right\rangle=\phi_{i}^{j}, \quad P_{i}^{j} P_{i+1}^{j} \rightarrow\left\langle P_{i}^{j} P_{i+1}^{j}\right\rangle \equiv \phi_{2 i}^{j} \\
S \rightarrow \sum_{i, j}\left[\alpha+\beta\left(1-P_{i}^{j}\right)\right]-\sum_{i, j}\left(P_{i}^{j+1}-P_{i}^{j}\right)^{2} \ln g+\frac{T_{0}}{2} \sum_{j=0}^{N} \sum_{i=1}^{M}\left[\left(\boldsymbol{x}_{i}^{j+1}-\boldsymbol{x}_{i}^{j}\right)^{2}+\left(\boldsymbol{y}_{i}^{j+1}-\boldsymbol{y}_{i}^{j}\right)^{2}\right] \\
\quad+\frac{T_{0}}{2} \sum_{j=1}^{N} \sum_{i=1}^{M}\left[\left(\boldsymbol{x}_{i+1}^{j}-\boldsymbol{x}_{i}^{j}\right)^{2} \phi_{i}^{j}+2 \boldsymbol{y}_{i}^{j 2}-2 \boldsymbol{y}_{i}^{j} \cdot \boldsymbol{y}_{i+1}^{j} \phi_{2 i}^{j}\right] \equiv S_{0}(\phi)
\end{gathered}
$$

To do this systematically, one can write $S \equiv S_{0}(\phi)+\Delta S(\phi)$ and treat $\Delta S(\phi)$ as a perturbation. Dropping $\Delta S$ is tantamount to the mean field approximation. From this point of view $\phi$ could be chosen to be anything, but it should be chosen to make the perturbative corrections as small as possible. Dropping $\Delta S$ decouples the coordinate path integral from the spin sum, so that the whole path integral factors into three parts $\mathcal{Z}=\mathcal{Z}_{x} \mathcal{Z}_{y} \mathcal{Z}_{s}$, or $\mathcal{F}=\mathcal{F}_{x}+\mathcal{F}_{y}+\mathcal{F}_{s}$ with

$$
\begin{aligned}
e^{-\mathcal{F}_{s}} & =\sum_{P_{i}^{j}=0,1} \exp \left\{-\sum_{i j}\left(\beta\left(1-P_{i}^{j}\right)+\left(P_{i}^{j+1}-P_{i}^{j}\right)^{2} \ln g+\kappa_{j}^{i} P_{i}^{j}\right)\right\}=\prod_{i} z_{s}\left(\kappa_{j}^{i}\right) \\
z_{s}\left(\kappa_{j}\right) & =\sum_{P^{j}=0,1} \exp \left\{-\sum_{j}\left(\beta\left(1-P^{j}\right)+\left(P^{j+1}-P^{j}\right)^{2} \ln g+\kappa_{j} P^{j}\right)\right\}
\end{aligned}
$$


Specializing to static sources $\kappa_{j}=\kappa$, the last sum can be thought of as the $N$ th power of the $2 \times 2$ matrix

$$
T=\left(\begin{array}{cc}
e^{-\beta} & g e^{-\kappa} \\
g e^{-\beta} & e^{-\kappa}
\end{array}\right)
$$

which has eigenvalues

$$
t_{ \pm}=e^{-(\beta+\kappa) / 2}\left[\cosh \frac{\kappa-\beta}{2} \pm \sqrt{\sinh ^{2} \frac{\kappa-\beta}{2}+g^{2}}\right]
$$

The contribution of each eigenstate of $T$ to $z_{s}$ is weighted by $t_{ \pm}^{N}$, and since $N \rightarrow \infty$ in the continuum limit, the + eigenstate will dominate:

$$
\prod_{i} z_{s}\left(\kappa^{i}\right) \sim C e^{N \sum_{i} \ln t_{+}\left(\kappa^{i}\right)}
$$

Since the coordinate integrations are decoupled from the spin sums at zeroth order in the mean field approximation, it follows (for static sources) that

$$
\begin{aligned}
\left\langle P_{i}^{j}\right\rangle_{0} & =-\frac{\partial}{\partial \kappa^{i}} \ln t_{+}\left(\kappa^{i}\right)=\frac{1}{2}\left(1-\frac{\sinh \left(\kappa_{i}-\beta\right) / 2}{\sqrt{\sinh ^{2}\left(\kappa_{i}-\beta\right) / 2+g^{2}}}\right) \\
\left\langle P_{i}^{j} P_{i+1}^{j}\right\rangle_{0} & =\left(\left\langle P_{i}^{j}\right\rangle_{0}\right)^{2}
\end{aligned}
$$

Then, making the choices $\phi_{i}^{j}=\left\langle P_{i}^{j}\right\rangle_{0} \equiv \phi_{i}$ and $\phi_{2 i}^{j}=\left\langle P_{i}^{j} P_{i+1}^{j}\right\rangle_{0}=\phi_{i}^{2}$ optimizes the perturbation theory in the sense that $\langle\Delta S\rangle_{0}=0$. These choices imply a linkage between $\phi_{i}$ and $\kappa_{i}$ which can be expressed as

$$
\kappa_{i} \approx \kappa_{0 i}(\phi) \equiv \beta+2 \ln \left\{\frac{g\left(1-2 \phi_{i}\right)}{2 \sqrt{\phi_{i}\left(1-\phi_{i}\right)}}+\sqrt{1+\frac{g^{2}\left(1-2 \phi_{i}\right)^{2}}{4 \phi_{i}\left(1-\phi_{i}\right)}}\right\}
$$

We have added the subscript 0 to $\kappa$ on the right of the last equation to emphasize that that relation between $\kappa$ and $\phi$, which we shall use to eliminate $\kappa$ in favor of $\phi$ in the spin part of the effective action, holds only at zeroth order and neglects the back-reaction of the coordinate fields-the essence of the mean field approximation. The remaining two terms in $\mathcal{F}$ are determined at zeroth order by

$$
\begin{aligned}
e^{-\mathcal{F}_{x}} & =\int \prod_{i j} d \boldsymbol{x}_{i}^{j} \exp \left\{-\frac{T_{0}}{2} \sum_{i j}\left[\left(\boldsymbol{x}_{i}^{j+1}-\boldsymbol{x}_{i}^{j}\right)^{2}+\phi_{i}\left(\boldsymbol{x}_{i+1}^{j}-\boldsymbol{x}_{i}^{j}\right)^{2}\right]\right\} \\
e^{-\mathcal{F}_{y}} & =\int \prod_{i j} d \boldsymbol{y}_{i}^{j} \exp \left\{-\frac{T_{0}}{2} \sum_{i j}\left[\left(\boldsymbol{y}_{i}^{j+1}-\boldsymbol{y}_{i}^{j}\right)^{2}+2\left(1-\phi_{i}^{2}\right) \boldsymbol{y}_{i}^{j 2}+\phi_{i}^{2}\left(\boldsymbol{y}_{i+1}^{j}-\boldsymbol{y}_{i}^{j}\right)^{2}\right]\right\}
\end{aligned}
$$




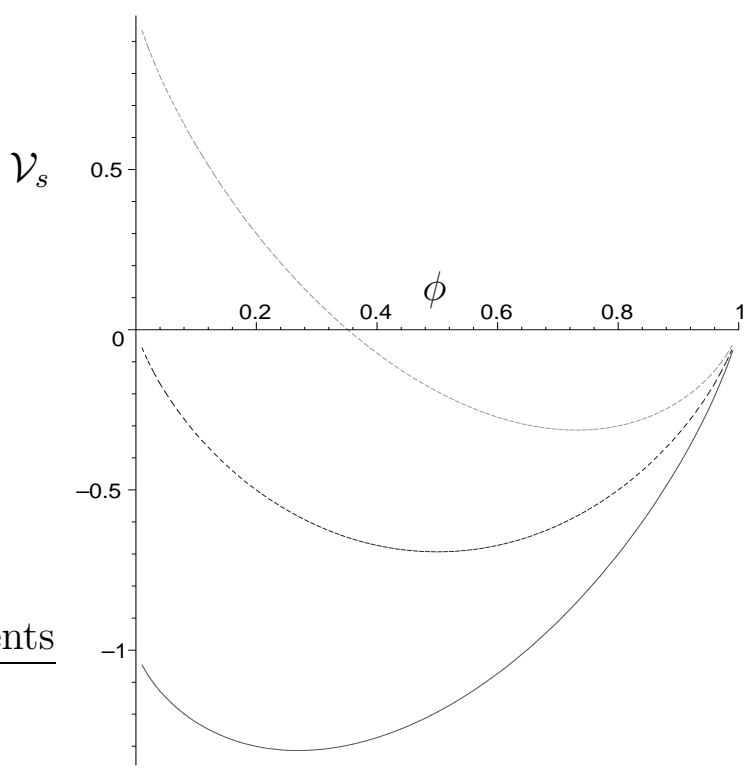

Figure 1: The spin contribution to the effective potential, showing the case of $g=1$ and $\beta=-1$ (lowest curve) $\beta=0$ (middle curve) and $\beta=1$ (highest curve).

Here we restrict attention to the planar (with respect to open string loops) evolution of the closed string, so that periodic boundary conditions are appropriate. Then by worldsheet translational invariance we can expect that the mean field in the system ground state is uniform over the worldsheet $\phi_{i}^{j}=\phi$. It is then sufficient to take a uniform source: $\kappa_{j}^{i}=\kappa$. We are also interested in the continuum limit $M, N \rightarrow \infty$, so the dominant contribution to the effective action, $\mathcal{A}=\mathcal{F}-M(N+1) \kappa \phi$, will be the bulk term $M(N+1) \mathcal{V}$ proportional to the area. The mean field will be determined by minimizing this term, i.e. by minimizing the effective potential $\mathcal{V}(\phi)=\mathcal{V}_{s}+\mathcal{V}_{x}+\mathcal{V}_{y}$. We next list the three contributions to $\mathcal{V}$ in the mean field approximation, including the $-\kappa \phi \approx-\kappa_{0}(\phi) \phi$ term in the spin contribution ${ }^{5}$.

$$
\begin{aligned}
\mathcal{V}_{s}= & \frac{\mathcal{F}_{s}\left(\kappa_{0}(\phi)\right)}{M(N+1)}-\kappa_{0}(\phi) \phi \rightarrow-\ln t_{+}-\kappa_{0}(\phi) \phi \\
= & \beta(1-\phi)+(1-2 \phi) \ln \left\{\frac{g(1-2 \phi)}{2 \sqrt{\phi(1-\phi)}}+\sqrt{1+\frac{g^{2}(1-2 \phi)^{2}}{4 \phi(1-\phi)}}\right\} \\
& -\ln \left\{\frac{g}{2 \sqrt{\phi(1-\phi)}}+\sqrt{1+\frac{g^{2}(1-2 \phi)^{2}}{4 \phi(1-\phi)}}\right\}
\end{aligned}
$$

\footnotetext{
${ }^{5}$ The technical details of their derivation can be gleaned from the appendices of $[18,19]$. Note that the condition $\kappa_{0}(\phi)=0$ determines a stationary point of $\mathcal{V}_{s}$ not $\mathcal{V}$. By minimizing the total $\mathcal{V}$ we take the back-reaction due to coordinate fluctuations into account in an average way.
} 


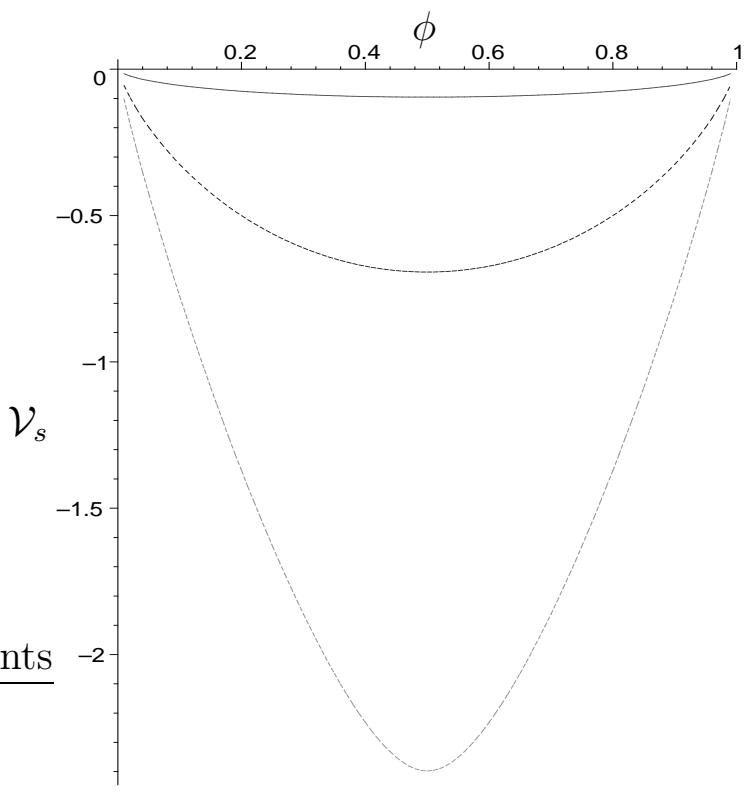

Figure 2: The spin contribution to the effective potential, showing the case of $\beta=0$ and $g=0.1$ (highest curve) $g=1$ (middle curve) and $g=10$ (lowest curve).

$$
\begin{aligned}
& \mathcal{V}_{x}=\frac{\mathcal{F}_{x}}{M(N+1)} \rightarrow(D-2) \int_{0}^{1} d x \sinh ^{-1}(\sqrt{\phi} \sin \pi x)=\frac{2(D-2)}{\pi} \sum_{n=0}^{\infty} \frac{(-)^{n} \phi^{n+1 / 2}}{(2 n+1)^{2}} \\
& \mathcal{V}_{y}=\frac{\mathcal{F}_{y}}{M(N+1)} \rightarrow(26-D) \int_{0}^{1} d x \sinh ^{-1} \sqrt{\frac{1}{2}\left(1-\phi^{2}\right)+\phi^{2} \sin ^{2} \pi x}
\end{aligned}
$$

Note that all of the dependence on the coupling $g$ and the counterterm parameter $\beta$ is carried by $\mathcal{V}_{s}$. As an illustration of this dependence we show a plot of $\mathcal{V}_{s}$ for $g=1$ and three different values for $\beta$ in Fig. 1 and for $\beta=0$ and three different values for $g$ in Fig. 2. Plots of $\mathcal{V}_{x}$ and $\mathcal{V}_{y}$ are shown separately in Fig. 3 , and combined (for the case $D=4$ ) in Fig. 4 . The non-monotonic behavior of this last graph is a direct consequence of the opposite monotonic behavior for Dirichlet and Neumann coordinates evident in Fig. 3. These curves will be the same for all values of $g$ and $\beta$. Of course the combined plot Fig. 4 will depend on $D$ which controls the relative weight of the coordinates with Neumann and Dirichlet boundary conditions. Finally in Figs. 5, 6, we plot the total effective potential $\mathcal{V}=\mathcal{V}_{x}+\mathcal{V}_{y}+\mathcal{V}_{s}$ for the same values of $\beta$ and $g$ used in Figs. 1, 2 .

In the mean field approximation, the value of $\phi$ controls the effective tension of the string. To see how, we write the effective action in the continuum limit:

$$
\mathcal{A}_{e f f} \rightarrow \frac{1}{2} \int d \tau d \sigma\left[\dot{\boldsymbol{x}}^{2}+\dot{\boldsymbol{y}}^{2}+T_{0}^{2}\left(\phi \boldsymbol{x}^{2}+\phi^{2} \boldsymbol{y}^{\prime 2}\right)+\frac{2}{a^{2}}\left(1-\phi^{2}\right) \boldsymbol{y}^{2}\right]
$$

For oscillations parallel to the D-branes (in the $\boldsymbol{x}$ directions) we have $T_{\|}^{e f f}=T_{0} \sqrt{\phi}$, and 

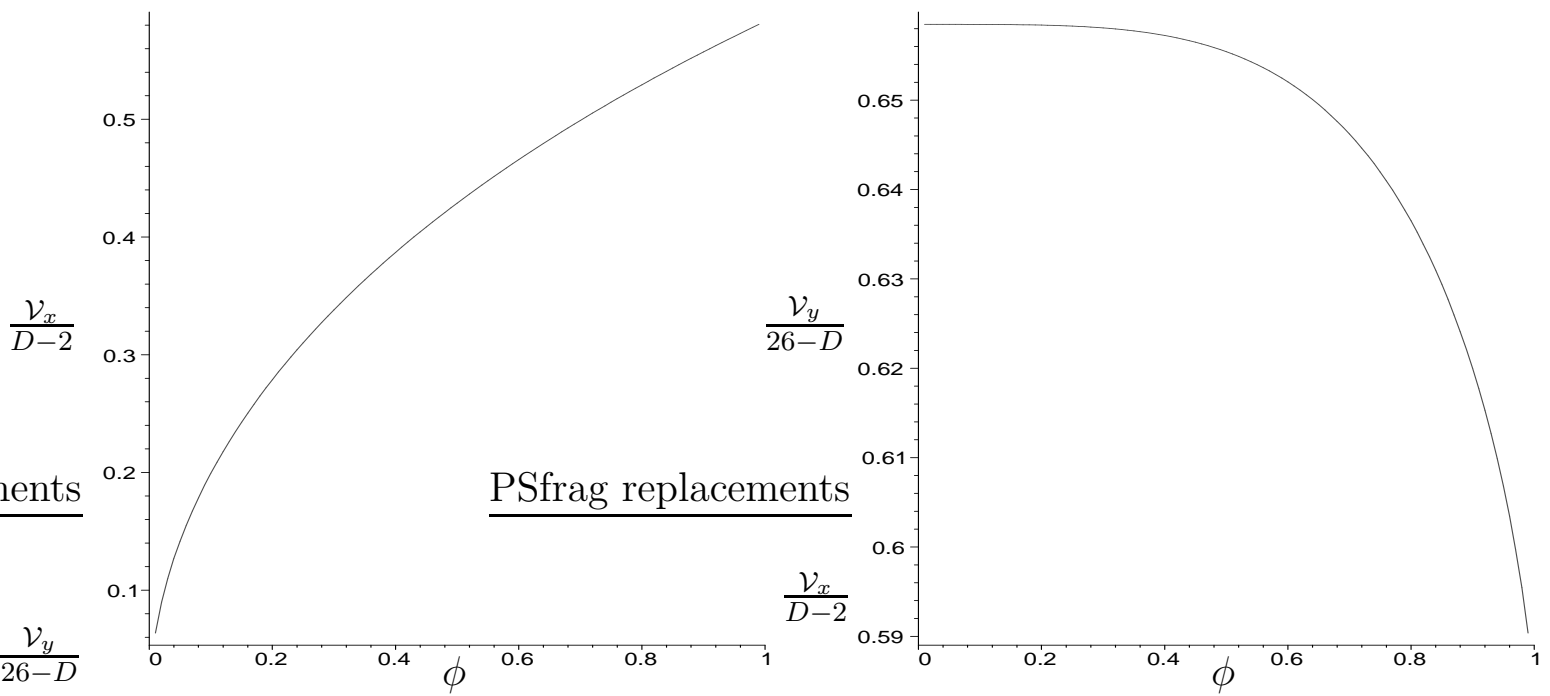

Figure 3: The coordinate contributions to the effective potential, Neumann on the left and Dirichlet on the right.

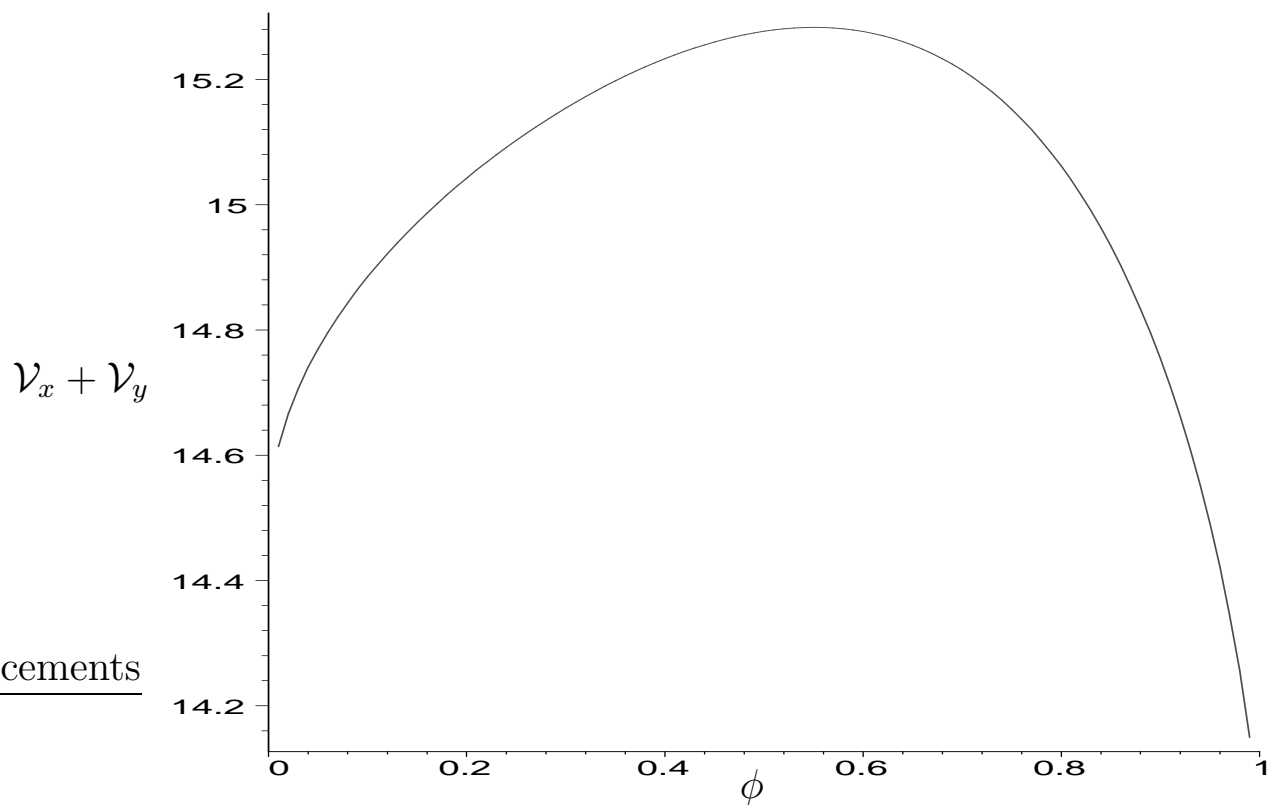

Figure 4: The total coordinate contribution to the effective potential, combined according to the case $D=4$. 
for oscillations perpendicular to the D-branes we have $T_{\perp}^{e f f}=T_{0} \phi$. The oscillations perpendicular to the brane also have an effective mass $m^{\text {eff }}=\sqrt{2\left(1-\phi^{2}\right)} / a$. For generic $\phi<1$ this mass is divergent in the continuum limit and would have the effect of suppressing oscillations perpendicular to the D-branes. For these oscillations to cost finite energy, would require $\phi=1+O\left(a^{2}\right)$ as $a \rightarrow 0$.

The plots of the effective potential in Figs. 5, 6 all show that both endpoints $\phi=0,1$ are local minima. The reason can be seen analytically from the endpoint behavior of $\mathcal{V}$. In the $\phi \rightarrow 0$ limit, $\mathcal{V}_{x}$ approaches zero with infinite positive slope, $\mathcal{V}_{y}$ approaches its limiting value with zero slope, and $\mathcal{V}_{s}$ approaches 0 with a negative infinite slope.

$$
\mathcal{V}_{x}^{\prime} \sim \frac{D-2}{\pi \sqrt{\phi}}, \quad \mathcal{V}_{y}^{\prime} \sim 0, \quad \mathcal{V}_{s}^{\prime} \sim-\ln \frac{1}{\phi}, \quad \text { for } \phi \rightarrow 0
$$

Clearly, $\mathcal{V}_{x}^{\prime}$ dominates as long as $D>2$, so $\mathcal{V}(\phi)$ rapidly increases as $\phi$ increases from 0 . At the other endpoint, $\phi=1, \mathcal{V}_{x}$ approaches its value at finite slope, $\mathcal{V}_{y}$ approaches its value at negative infinite slope, and $\mathcal{V}_{s}$ approaches its value at positive infinite slope.

$$
\mathcal{V}_{x}^{\prime} \sim \frac{D-2}{4}, \quad \mathcal{V}_{y}^{\prime} \sim-\frac{26-D}{2 \pi} \ln \frac{1}{1-\phi}, \quad \mathcal{V}_{s}^{\prime} \sim \ln \frac{1}{1-\phi}, \quad \text { for } \phi \rightarrow 1
$$

Here $\mathcal{V}_{y}, \mathcal{V}_{s}$ are comparable and we conclude that

$$
\mathcal{V}^{\prime} \sim-\frac{26-D-2 \pi}{2 \pi} \ln \frac{1}{1-\phi}, \quad \text { for } \phi \rightarrow 1
$$

and $\mathcal{V}$ will increase from its value as $\phi$ decreases from 1 as long as $D<26-2 \pi$. It follows that, for small to moderate coupling, only the endpoints $\phi=0,1$ are candidate minima of the effective potential. Which one is actually lower in energy is controlled by the value of $\beta$, which is not known a priori for general $g$. From the free open string calculation we know that $\beta \approx-3.91+O\left(g^{2}\right)$ for $D=4$. For a value of $\beta$ this negative, $\phi=0$ is favored over $\phi=1$ by a very wide margin for small to moderate couplings. Keeping $\beta$ fixed at this value, one would have to go to $g>7$ for a minimum with $0<\phi<1$ to develop. Since the "bare" one loop correction comes entirely from second order perturbation theory, it should lower the zero coupling energy and hence require the $O\left(g^{2}\right)$ contribution to $\beta$ to be positive, making it a little less negative. In any case, we can safely say that mean field theory predicts that the effective string tension will vanish in the system ground state at very weak coupling $g \ll 1$.

For $g$ sufficiently large at fixed $\beta$, a local minimum in the effective potential develops at some $\phi_{0}$ between 0 and 1 . However, at the critical coupling where $\mathcal{V}^{\prime \prime}\left(\phi_{0}\right)$ vanishes, this local minimum has higher energy than one or both of the two endpoint minima, so this minimum initially describes a metastable phase. Near the critical point this metastable phase would support finite energy spin waves, signifying the emergence of a new Liouville-like degree of freedom on the worldsheet. But eventually for larger coupling, this new minimum could become a global minimum and the system ground state would support a finite string tension. However, since we do not know $\beta$ for $g=O(1)$, we cannot rule out the possibility that $\beta$ becomes more and more negative as $g$ grows. If this happens the local minimum 


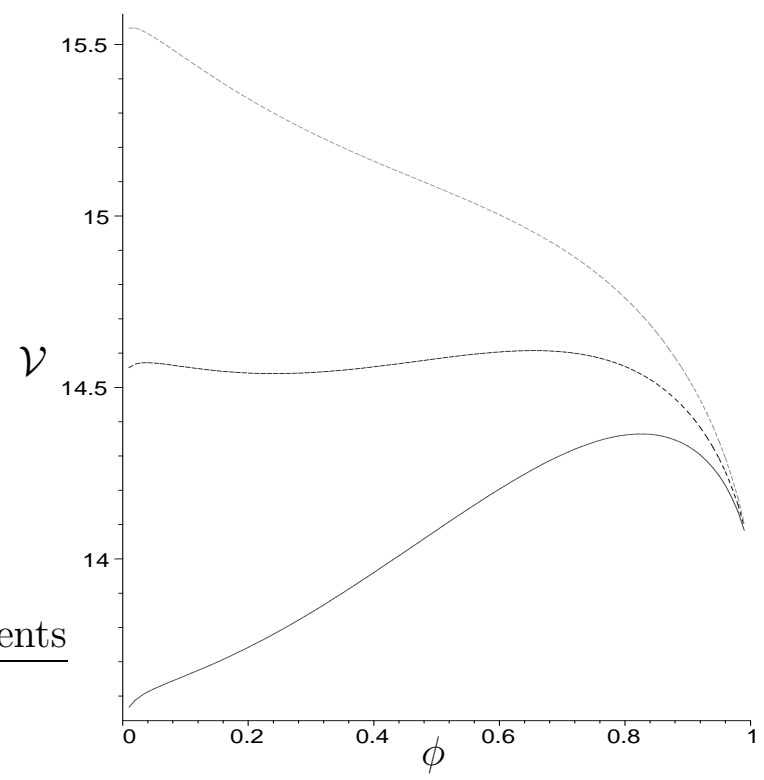

Figure 5: The effective potential, showing the case of $g=1$ and $\beta=-1$ (lowest curve) $\beta=0$ (middle curve) and $\beta=1$ (highest curve).

just described may never become a global one. Thus even in the mean field approximation, the jury is still out on the question of whether our lattice model of the bosonic string will actually support a finite string tension.

\section{Discussion and Conclusions}

In this article we have shown how to extend the lattice worldsheet formalism for the bosonic string to allow for D-branes, and we have applied a mean field approximation to the resulting lattice model. Although the mean field analysis allowed us to map out the possibile phases of the system, our a priori ignorance of the value of $\beta(g)$ leaves us uncertain about which phase is actually realized when $g \geq O(1)$. However, the mean field analysis is uneqivocal about the weak coupling phase: in it the mean field $\phi=0$ and the string tension is quenched to zero.

There are many levels at which our results must be regarded as provisional. First is the question: does the lattice formalism accurately represent the bosonic string perturbation theory? The answer is yes only if the bulk and boundary counterterms we have allowed for are sufficient to absorb all Lorentz covariance violating artifacts due to the lattice cutoff. The evidence for this is so far very meager. These counterterms suffice to render the spectrum and tree scattering amplitudes Lorentz covariant. At one loop, we have analyzed the open string propagator and shown that for this specific one loop process the counterterms suffice. It should not be too difficult to check this conclusion for higher point one loop amplitudes, but this has not yet been done. We have no information on this issue at two loops and 


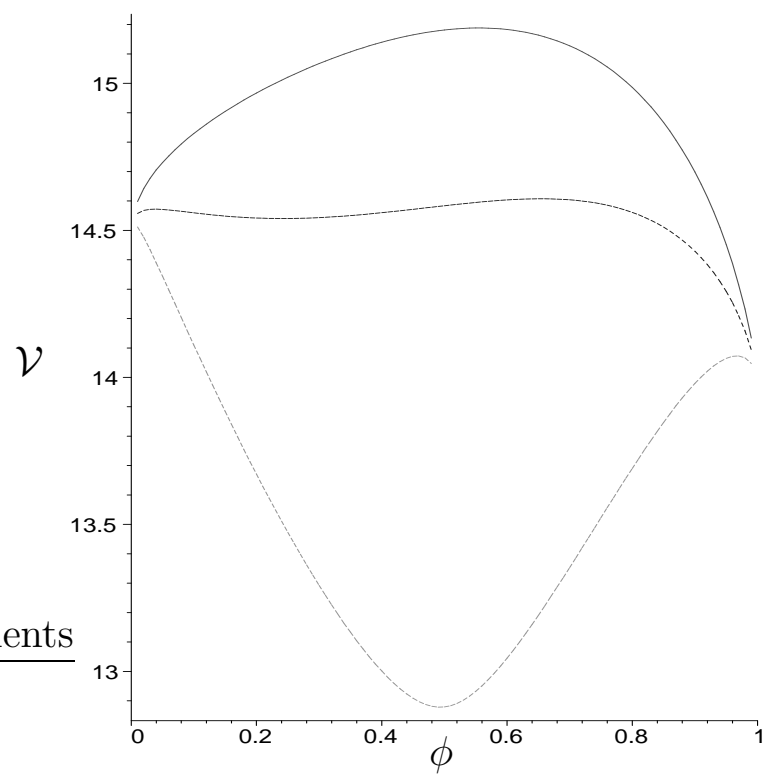

Figure 6: The effective potential, showing the case of $\beta=0$ and $g=0.1$ (highest curve) $g=1$ (middle curve) and $g=10$ (lowest curve).

beyond. Clearly more investigation of this quesiton is called for.

Even if the worldsheet lattice system is not Lorentz covariant because of the need for more complicated counter-terms, it remains a well-defined two dimensional system of scalar fields interacting with an Ising spin system. The physics of this system can be analyzed in its own right. In this article we have begun this analysis within the mean field approximation. The analysis suggests that the system exhibits three distinct phases: a phase with zero effective string tension $(\phi=0)$, a phase with maximal effective tension $T_{0}(\phi=1)$ and a disordered spin phase, only stable at sufficiently large coupling, with reduced effective tension $T_{0} \sqrt{\phi}$ $(0<\phi<1)$. This intermediate phase could potentially support a meaningful infinite tension limit in which the effective tension stays finite. In such a limit the planar string diagrams would go over into planar quantum field theory diagrams, and one might gain insight into the large $N$ limit of certain matrix field theories. All of these conclusions depend on the validity of the mean field approximation, which by its very nature is somewhat dubious. But there are other approaches to analyzing this system. In particular, Monte Carlo methods seem particularly apt, since the path integrand is positive definite and local. Such an analysis to test the mean field conclusions would be very welcome.

Finally, we have to recognize that the tachyon in the bosonic open string theory obscures the meaning of the open string loop expansion our model is meant to represent. By itself the tachyon could simply mean that the system is being studied in an unstable vacuum, and it might disappear once a stable vacuum is found. Since our lattice model is a perfectly welldefined physical system, its physics could provide information about the correct stable ground state. Indeed, the indication, from the mean field approximation at weak coupling, that the 
string tension is quenched to zero could be the ultimate fate of the tachyon instability: the bosonic open string would then be unstable to decaying into an infinite number of string bits [20]. If this is the case, the open bosonic string theory would not be a good starting point for understanding large $N$ gauge theory. However, the even G-parity sector of the NeveuSchwarz model is free of open string tachyons and provides a more promising approach to large $N$ QCD $[13,14]$. This possibility makes the extension of the lightcone lattice worldsheet formalism to include Grassmann variables a particularly desirable next step.

Acknowledgments: I would like to thank Peter Orland and Nati Seiberg for helpful discussions. I would also like to acknowledge the hospitality of the School of Natural Sciences at the Institute for Advanced Study, where this work was carried out. This research was supported in part by the Ambrose Monell Foundation and in part by the Department of Energy under Grant No. DE-FG02-97ER-41029.

\section{References}

[1] G. 't Hooft, Nucl. Phys. B72 (1974) 461.

[2] J. Maldacena, Adv. Theor. Math. Phys. 2, 231 (1998), hep-th/9711200.

[3] R. Giles and C. B. Thorn, Phys. Rev. D 16 (1977) 366.

[4] P. Goddard, Nuovo Cim. A 4 (1971) 349; A. Neveu and J. Scherk, Nucl. Phys. B 36 (1972) 317.

[5] M. B. Green and N. Seiberg, Nucl. Phys. B 299 (1988) 559.

[6] K. Bardakci and C. B. Thorn, Nucl. Phys. B 626 (2002) 287 [arXiv:hep-th/0110301]; C. B. Thorn, Nucl. Phys. B 637 (2002) 272 [Erratum-ibid. B 648 (2003) 457] [arXiv:hepth/0203167]; S. Gudmundsson, C. B. Thorn and T. A. Tran, Nucl. Phys. B 649 (2003) 3 [arXiv:hep-th/0209102].

[7] J. Dai, R. G. Leigh and J. Polchinski, Mod. Phys. Lett. A 4 (1989) 2073.

[8] A. Neveu and J. H. Schwarz, Nucl. Phys. B 31 (1971) 86.

[9] A. Neveu, J. H. Schwarz and C. B. Thorn, Phys. Lett. B 35 (1971) 529.

[10] P. Ramond, Phys. Rev. D 3 (1971) 2415.

[11] A. Neveu and J. H. Schwarz, Phys. Rev. D 4 (1971) 1109; C. B. Thorn, Phys. Rev. D 4 (1971) 1112.

[12] F. Gliozzi, J. Scherk and D. I. Olive, Phys. Lett. B 65, 282 (1976); Nucl. Phys. B 122 (1977) 253. 
[13] C. B. Thorn, Phys. Rev. D 78 (2008) 085022 [arXiv:0808.0458 [hep-th]].

[14] C. B. Thorn, Phys. Rev. D 78 (2008) 106008 [arXiv:0809.1085 [hep-th]].

[15] P. Goddard, J. Goldstone, C. Rebbi and C. B. Thorn, Nucl. Phys. B 56 (1973) 109.

[16] P. Orland, Nucl. Phys. B 278 (1986) 790.

[17] K. Bardakci, Nucl. Phys. B 746 (2006) 136 [arXiv:hep-th/0602131].

[18] K. Bardakci and C. B. Thorn, Nucl. Phys. B 652 (2003) 196 [arXiv:hep-th/0206205].

[19] C. B. Thorn and T. A. Tran, Nucl. Phys. B 677 (2004) 289 [arXiv:hep-th/0307203].

[20] C. B. Thorn, Invited talk to 1st Int. A.D. Sakharov Conf. on Physics, Moscow, U.S.S.R., May 27-31, 1991; Sakharov Memorial Lectures in Physics: Proceedings, Ed. L.V. Keldysh and V.Ya. Fainberg, Nova Science (1992), pp.447-454; arXiv:hep-th/9405069. 